\title{
Temporal and Spatial Variations of Cosmogenic Radionuclide Production Rates in Chondrites During Their Passage Through the Inner Heliosphere
}

\author{
Galina Ustinova*, Victor Alexeev \\ Laboratory of Meteoritics and Cosmochemistry, Vernadsky Institute of Geochemistry and Analytical Chemistry, Russian Academy of \\ Sciences, Moscow, Russia
}

Email address:

ustinova@dubna.net.ru (G. Ustinova)

${ }^{*}$ Corresponding author

\section{To cite this article:}

Galina Ustinova, Victor Alexeev. Temporal and Spatial Variations of Cosmogenic Radionuclide Production Rates in Chondrites During Their Passage Through the Inner Heliosphere. American Journal of Physics and Applications. Vol. 8, No. 3, 2020, pp. 29-39.

doi: 10.11648/j.ajpa.20200803.11

Received: April 20, 2020; Accepted: May 6, 2020; Published: June 9, 2020

\begin{abstract}
To study radiation environment in the interplanetary space, cosmogenic radionuclides in meteorites, the production rates of which are in direct proportionality to the intensity of cosmic rays, are used. The contents of cosmogenic radionuclides of different half-lives $T_{1 / 2}$, measured in 42 stony meteorites (chondrites) having sequentially fallen onto the Earth during the period of 1959-2016, are analyzed. They are accumulated by the galactic cosmic rays (GCRs) along the orbits of the chondrites before their falls onto the Earth at some average heliocentric distances, depending on the size of the chondrite orbit and on $T_{1 / 2}$ of the radionuclide. The comparison with the calculated production rates of radionuclides in the identical chondrites for isotropic irradiation by the GCRs at $\sim 1 \mathrm{AU}$ is demonstrated. The calculations are based on the stratospheric balloon monthly data on the GCR intensity [1] for the periods of accumulation of each radionuclide in each chondrite. The dependence of production rates of the radionuclides of different half-lives upon the GCR variations in the heliosphere is studied. The obtained long set of homogeneous data on cosmogenic radionuclide production rates in consecutively fallen chondrites provides the unique information on the space-time continuum of the cosmogenic radionuclide production rates and their variations over a long-time scale, which could be useful in the correlative analyses of processes in the inner heliosphere and, thus, in the forecast of radiation situation, which is important for the predicted manned flights.
\end{abstract}

Keywords: Chondrites, Cosmogenic Radionuclides, Inner Heliosphere, Solar Modulation, Cosmic Rays

\section{Introduction}

It is natural that orbits of the meteorites (in particular, the stony meteorites - chondtrites) that could fall onto the Earth have perihelia $q$ near $\sim 1 \mathrm{AU}$, which is confirmed by the data of the world bolide's net for photographing meteoritic falls [2] as well as by the numerous results of the thermoluminescence study of the fallen samples [3].

On the other hand, the information on the position of aphelia $Q$ is less definite, because of the uncertainty of chondrite velocity. However, the velocity range for the chondrites that could fall onto the Earth is restricted by the minimum geocentric velocity $v_{\infty} \sim 11.2 \mathrm{~km} / \mathrm{s}$ (defined by the terrestrial attraction) and by its maximum value of $v_{\infty} \sim 22$ $\mathrm{km} / \mathrm{s}$ (defined by the atmospheric selection), above which the celestial body is completely broken down $[4,5]$. This means that the aphelia of most of the fallen chondrites lay inside the Jupiter orbit, i.e. at $Q \leq 5 \mathrm{AU}$ of the inner heliosphere [6, 7]

The motion of chondrites, like other cosmic bodies, obeys Kepler's laws, so that their orbits in the coordinates $r(t)$ are described by the Kepler equations (see, for example, [8]):

$$
\left\{\begin{array}{l}
r=a(1-e \cos E) \\
t=\frac{T}{2 \pi}(E-e \sin E)
\end{array}\right.
$$

where $a$ is the semi-major axis, $e$ is the eccentricity, $E$ is the eccentric anomaly, and $T$ is the orbital period. When $t=0$, then $r=q$, i.e., the perihelion, and at $t=T / 4 r=\tilde{r}_{o}$, i.e., the 
average heliocentric distance of the chondrite orbit.

During the motion of a chondrite along the orbit, cosmogenic radionuclides are generated in cosmic-ray induced nuclear reactions on all the elements of the chondrite composition $[9,10]$. Due to the successful development of accelerator mass spectrometry (e.g., [11]) and highly sensitive (often underground) gamma spectrometry with germanium detectors (HPGe) [12, 13], a lot of cosmogenic radionuclides of different half-lives $T_{1 / 2}$ (e.g., from ${ }^{24} \mathrm{Na}$ $\left(T_{1 / 2}=15 \mathrm{~h}\right),{ }^{48} \mathrm{~V}\left(T_{1 / 2}=16\right.$ days $)$, and ${ }^{22} \mathrm{Na}\left(T_{1 / 2}=2.6\right.$ years $), \ldots$ up to ${ }^{26} \mathrm{Al}\left(T_{1 / 2}=0.717 \mathrm{Ma}\right),{ }^{53} \mathrm{Mn}\left(T_{1 / 2}=3.7 \mathrm{Ma}\right)$ and ${ }^{40} \mathrm{~K}$ $\left.\left(T_{1 / 2}=1.248 \mathrm{Ga}\right)\right)$ became available for study in the fallen chondrites (even in milligram samples).

The cosmogenic radionuclide contents (about 80\%), measured at the time of fall of the chondrites to the Earth, is accumulated in them for $\sim 1.5 T_{1 / 2}$ of the radionuclides before falling. Solving the system of Kepler's equations for the motion of a chondrite along the orbit, one can single out a part of the orbit where the average content of each radionuclide is accumulated before a chondrite fall. For example, the long-lived ${ }^{26} \mathrm{Al}$ is formed during many revolutions of a chondrite around the Sun under the action of GCR medium-intensity for $\sim 1$ million years at an average heliocentric distance of the chondrite orbit. The measured content of radionuclides with $T_{1 / 2} \leq T$ is accumulated in course of $\sim 1.5 T_{1 / 2}$ of the radionuclides in the last part of the chondrite orbit before its fall to the Earth under the action of GCR medium-intensity for that time interval, which is natural to correlate with the average heliocentric distance of the chondrite at $\sim 0.75 T_{1 / 2}$. It is obvious that the short-lived radionuclides are formed under the action of GCR medium intensity near the Earth at the fall of the chondrite.

For instance, the orbits $r(t)$ of the Pribram and Lost City chondrites (the first chondrites of known orbits) are demonstrated in Figure 1. One may see there that in the Pribram chondrite the measured amount of ${ }^{54} \mathrm{Mn}$ was produced at 2.96 AU during 450 days and that of ${ }^{22} \mathrm{Na}$ was produced at $4.05 \mathrm{AU}$ during $\sim 4$ years before the fall of this chondrite on April 7, 1959. Similarly, in the Lost City chondrite the measured amount of ${ }^{54} \mathrm{Mn}$ was produced at 1.93 AU during 450 days and that of ${ }^{22} \mathrm{Na}$ was produced at 1.30 AU during $\sim 4$ years before the fall of this chondrite on January 1, 1970. It is interesting that ${ }^{60} \mathrm{Co}$ in both the chondrites was accumulated at the $\sim 1.7$ AU before $\sim 8$ years of their fall onto the Earth in 1959 and 1970, respectively. At the same distance $(\sim 1.7 \mathrm{AU})$ the measured amount of ${ }^{46} \mathrm{Sc}$ was accumulated in Pribram, while that in Lost City was produced at $\sim 1.3 \mathrm{AU}$. In conditions of the secular equilibrium, the produced quantity of a cosmogenic radionuclide equals to its production rate by cosmic rays.

As it may be seen well, measuring and analyzing cosmogenic radionuclides with different $T_{1 / 2}$ in permanently falling chondrites provide us with a powerful instrument for the subtle inspection of cosmogenic radionuclide production rates at different heliocentric distances of the inner heliosphere during different solar cycles over a long-time scale. Why is it necessary?

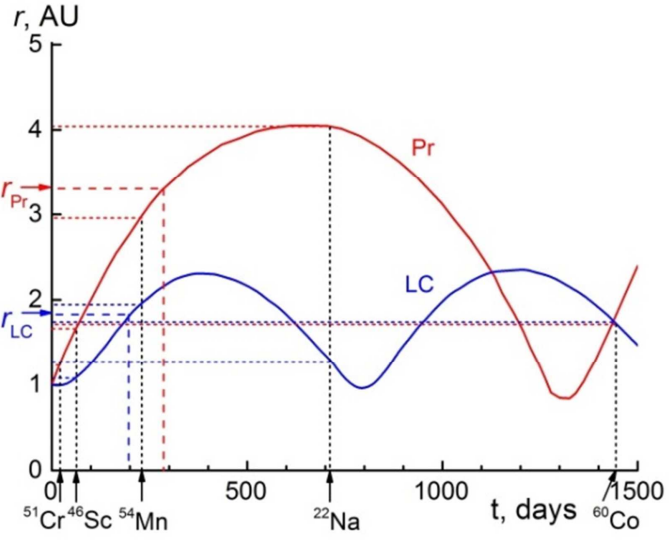

Figure 1. Dependence of heliocentric distance $r$ on time t for the chondrites Pribram (Pr) and Lost City ( $L C)$ up to their striking to the Earth at $t=0$. Dates of the average effective productions of the radionuclides $\left(\sim 0.75 T_{1 / 2}\right)$ are marked on $x$-axis, and corresponding average heliocentric distances for their production are given on y-axis. $\left(r_{P r}\right.$ and $r_{L C}$ are respective average heliocentric distances of the chondrite orbits).

Towards the GCRs isotropically entering the heliosphere from the outer space, the Sun radiates solar wind (protons and electrons with $E<10 \mathrm{eV}$ ) and solar cosmic rays (SCR), mainly solar protons with $E>10 \mathrm{MeV}$, which, while weakening proportionally to the distances squared, propagate up to the dynamic equilibrium boundary with interstellar gas at $\geq 100$ AU. This leads to a gradual decrease in the intensity of the incoming GCRs, on account of their modulation by the total volume of the solar wind, which is characterized by small $\sim 2-4 \%$ /AU GCR gradients $(E \geq 100 \mathrm{MeV})$ [14]. However, such a constant uniformity is inconsistent with dynamic processes and configurations of magnetic fields in the heliosphere. In accordance with the 11-year variations of solar activity, the movement of the solar wind and SCRs is primarily radial but, due to the rotation of the Sun, their paths are gradually bent, twisting in a spiral of Archimedes, and approximately by $\sim 5 \mathrm{AU}$ they become practically azimuthal [15]. The magnetic fields of the solar wind frozen into the plasma create, while twisting, insurmountable barriers for the penetration of GCRs to the Sun; i.e., they practically sweep them from the heliosphere. The higher is the solar activity, the lower is the GCR intensity on the Earth. Thus, one may suggest that, in addition to the GCR modulation conditioned by the total volume of the solar wind, during high solar activity (due to the worse conditions for the diffusion of GCRs in azimuthal fields than in radial ones) by 2-5 AU from the Sun the periodically more efficient GCR modulation occurs, which is conditioned by the increased turbulence of magnetic fields and increased density of magnetic irregularities, effectively scattering GCRs [16], and creating an additional gradient of their intensity to a small gradient with a calm solar wind. When solar activity declines, nonstationary processes weaken, and the residual modulation is determined again mainly by the total volume of the solar wind.

However, such an important and dynamically disturbed region of the inner heliosphere is not yet practically 
investigated. Of course, the 11-year cycle of the GCR solar modulation at $\sim 1 \mathrm{AU}$ is studied well enough due to the numerous direct measurements in stratospheric [1] and ground-based experiments [17], and especially, due to the PAMELA satellite experiments (e.g., [18]). But what is going on the other heliocentric distances of that problem range of the inner heliosphere? Certainly, there were GCR measurements during the flights of spacecrafts Mariner-4,-5 in 1964-1965, Pioneer-10,-11 in 1972-1976, and Voyager-1,2 in 1984-1986 [19-21], which indicate to a continuous and uniform increase in the GCR intensity with the distance from the Earth up to its alignment with the GCR intensity in space at the boundary of the heliosphere [22]. Meanwhile, it should be noted that the above spacecraft launches were made during periods of solar activity minima of 19, 20, and 21 solar cycles, when there was no modulation in the inner heliosphere and the GCR gradients were small. In the years of the development of solar maxima, the spacecrafts were already outside the inner heliosphere, where the adiabatic cooling, i.e. deceleration of the solar wind and its distribution throughout the permanently increasing volume of steadily decreasing density of the magnetic irregularities, led to the gradual weakening of their influence, so that only small GCR gradients were recorded again. Firstly, direct GCR measurements within $\sim 5 \mathrm{AU}$ from the Sun and variations of the GCR gradients, depending on solar activity, were recorded only after the launch of the Ulysses spacecraft at its maximum in 1990 [23]. A tenfold increase of the integral gradients of protons of $E>2 \mathrm{GeV}$ was registered for the solar activity maximum in $1998-2001(5.3 \% / \mathrm{AU})$ in comparison with those $(0.5 \% / \mathrm{AU})$ for the solar activity minimum in 1994-1996 [24].

It is clear that scant data of such rare and high-cost missions cannot reveal regularities of the dynamical processes in the inner heliosphere and their variations with time. In this connection, cosmogenic radionuclide production rates in chondrites with different orbits, permanently falling onto the Earth attract our attention. Indeed, on account of direct proportionality to the GCR intensity, just the cosmogenic radionuclide production rates in the fallen chondrites might inspect the radiation situation at different heliocentric distances of the interplanetary space for the different stages of different solar cycles over a long-time scale.

\section{Cosmogenic Radionuclide Production Rates in Chondrites}

In cosmic bodies, cosmogenic radionuclides are formed by galactic and solar cosmic rays, but in chondrites, due to their ablation during the passage through the terrestrial atmosphere, only the effects of GCR irradiation with the energy of $>100$ $\mathrm{MeV}$ remain. The SCR effects with energies $>20 \mathrm{MeV}$, observed in lunar samples, appear only in layers of $<2 \mathrm{~cm}$ from the surface, which are lost in chondrites during ablation [25]. According to observations in the Earth's atmosphere (i.e., at $1 \mathrm{AU}$ ) [26], protons with $E>100 \mathrm{MeV}$, which are generated in the most powerful (but local in time) solar flares, also give a negligible global effect, which decreases in chondrites but is still proportional to $\sim \tilde{r}_{0}^{2}$. It is a happy case that just such GCR integral fluxes $(E>100 \mathrm{MeV})$ can be determined in the monthly balloon experiments of The Lebedev Physical Institute, RAS in the stratosphere since 1957 [1], the use of which allows us to calculate the cosmogenic radionuclide production rate in the fresh-fallen chondrites, i.e. at $\sim 1$ AU.

The modeling of nuclear reactions under the isotropic action of cosmic rays in cosmic bodies can be performed by applying an efficient quantitative analytical method of using cosmogenic radionuclides as natural detectors of cosmic rays $[9,27-30]$. This is based on a cascade-evaporation model, which has been elaborated in detail for the description of cosmogenic radionuclide production rates for a specific spectrum and a given intensity of cosmic rays at any depth for cosmic bodies of any size and composition. The analytical expressions for the nuclear cascade development up to the $3^{\text {rd }}$ stage of the process are presented. Since the whole problem is expressed in an analytical form, the method can be easily used in any specific case, i.e., for estimation of the content of any cosmogenic radionuclide produced by cosmic rays of any energy spectrum and intensity at any depth of a cosmic body of any size and composition (for instance, meteorites of approximately spherical shape are isotropically irradiated in $4 \pi$-geometry, while the surface of the Moon is irradiated in $2 \pi$-geometry). The precision of this analytical method was proved in the direct experiment on $4 \pi$ irradiation of a spherical thick iron target, rotating in two plates, with a $660 \mathrm{MeV}$ proton beam of the Dubna synchrocyclotron [31].

The production rate of $i$-radionuclide in a stony meteorite may be approximated in the general form as

$$
H_{i}(R, r) \sim\left[I_{p}(R, r) \sum_{j=1}^{n} \frac{N}{A_{j}} m_{j} \bar{\sigma}_{i j}^{p}+\sum_{s} I_{s}(R, r) \sum_{j=1}^{n} \frac{N}{A_{j}} m_{j} \bar{\sigma}_{i j}^{s}+\sum_{t} I_{t}(R, r) \sum_{j=1}^{n} \frac{N}{A_{j}} m_{j} \bar{\sigma}_{i j}^{t}+\ldots\right]
$$

where $N$ is the Avogadro number, $I_{p, s, t}(R, r)$ is the integral flux of primary, secondary and tertiary particles in a cosmic body of radius $\mathrm{R}$ at the depth $\mathrm{r}$ from the center, $\overline{\boldsymbol{\sigma}}_{i j}^{p, s, t}$ are the mean-weighted production cross sections of $i$-radionuclide from $j$-target of mass number $A_{j}$ for primary, secondary and tertiary particles, which may be expressed in the form (e.g., in the case of primary particles)

$$
\bar{\sigma}_{i j}^{p}=\int \frac{\sigma_{i j}^{p}(E) F^{p}(E)}{F^{p}(E)} d E
$$

where $m_{j}$ is the abundance of $\mathrm{j}$-target element in the meteorite. The incident integral GCR intensity $I_{p, s, t}(R, r)$ may be taken out of the brackets, and then the radionuclide production rate $H_{\tilde{r}}$ at the average heliocentric distance $\tilde{r}$ is proportional to the integral GCR intensity $I_{\tilde{r}}(>E)$ at the distance $\tilde{r}$ :

$$
H_{\tilde{r}}=I_{\tilde{r}}(>E) K_{\tilde{r}}\left(R, r, \sigma^{p, s, t}, m\right)
$$

The production rate $H_{\tilde{r}}$ is equal to the average content of a 
radionuclide at the average heliocentric distance $\tilde{r}$ of its accumulation, measured at the moment of a chondrite fall (see Introduction). Similarly, $H_{\oplus}$ is the radionuclide content in the same chondrite, calculated using the GCR flux obtained from the stratospheric data [1], and representing the production rate near the Earth:

$$
H_{\oplus}=I_{\oplus}(>E) K_{\oplus}\left(R, r, \sigma^{p, s, t} m\right)
$$

As follows from the Eq. (2), the accuracy of calculation of cosmogenic radionuclide production rates in each chondrite depends not only on the direct proportionality to proper GCR intensity, but also on the individual chemical composition of the chondrite as well as on its preatmospheric size $(R)$ and screening depth $(r)$ of the investigated samples.

Under the conditions of GCR isotropic irradiation of chondrites in the interplanetary space, the content of radionuclides with $T_{1 / 2} \leq T$, measured at the time of their fall, is accumulated in course of the time interval of $\sim 1.5 T_{1 / 2}$ of the radionuclides in the last part of the chondrite orbit before its fall to the Earth, which is natural to correlate with the average heliocentric distance of the chondrite for that time interval (as it was already noted before). The comparison of the measured radionuclide contents $H_{\tilde{r}}$ with their calculated contents $H_{\oplus}$ in identical chondrites at the GCR intensity of 1 AU (according to the available data of stratospheric measurements) allows one to determine the integral gradient of cosmogenic radionuclide production rates (if those exist)

$$
G_{\tilde{r}}=\frac{H_{\tilde{r}} / H_{\oplus}-1}{\tilde{r}-1} \cdot 100 \%
$$

Its direct proportionality to the integral GCR $(E>100$ $\mathrm{MeV}$ ) gradients

$$
G_{\tilde{r}}(>E)=\frac{I_{\tilde{r}}(>E) / I_{\oplus}(>E)-1}{\tilde{r}-1} \cdot 100 \%
$$

is natural, which allows us to obtain some indirect information on the GCR distribution and variations at different heliocentric distances at different periods of time in the Solar system for the long-time scale over the last $\sim 1 \mathrm{Myr}$. Such a rare possibility is not yet available for direct measurements in the interplanetary space, moreover, in the volumes that constantly fallen chondrites can provide.

\section{Preatmospheric Sizes of Chondrites and Screening Depths of the Samples}

A significant problem in the use of cosmogenic radionuclides in meteorites as cosmic-ray detectors is the estimation of the preatmospheric size of meteorites and the depth of the samples under study. Just the analytical method of calculation of cosmogenic radionuclide depth distributions in isotropically irradiated cosmic bodies of different size and composition allowed us to elaborate about 10 independent approaches to the problem and to find and use the ones being the most sensitive to the size of chondrites and screening depth of the samples $[9,32]$ As it is currently conventional, the most sensitive methods are based either on the content of the ${ }^{60} \mathrm{Co}$ neutron capture products $[32-34]$, or on the ratio ${ }^{60} \mathrm{Co} /{ }^{26} \mathrm{Al}[35]$ in combination with measuring the density of tracks of $\mathrm{VH}$-nuclei $(23<Z<29)$ in olivine and pyroxene crystals of chondrites, where they are recorded during the cosmic ray irradiation $[5,36]$. Below, as an example, the procedure is demonstrated in the case of the Ash Creek L6 chondrite that fell in the morning of February 15. 2009 in Texas, US.

Using the analytical method, the modeling of ${ }^{60} \mathrm{Co}$ radionuclide production rates in the Ash Creek L6 chondrite is carried out (see Figure 2). In the cascade process initiated by the GCR irradiation, ${ }^{60} \mathrm{Co}$ radionuclide is produced by thermal and resonance neutrons in the reaction of ${ }^{59} \mathrm{Co}(\mathrm{n}, \gamma){ }^{60} \mathrm{Co}$ [33]. In the calculation, the data on ${ }^{59} \mathrm{Co}$ content in L6 chondrites (0.06 weight. \%) [37] as well as the average GCR intensity $I(E>100 \mathrm{MeV}) \sim 0.2423 \mathrm{~cm}^{-2} \mathrm{~s}^{-1}$ ster $^{-1}$ during $\sim 8$ years $\left(\sim 1.5 \mathrm{~T}_{1 / 2}{ }^{59} \mathrm{Co}\right)$ before the fall of the chondrite to the Earth, according to stratospheric measurements of [1], were used.

It is well seen in Figure 2 that the measured content of ${ }^{60} \mathrm{Co}$ in the Ash Creek chondrite (the cross corresponding to $41 \pm 5 \mathrm{dpm} / \mathrm{kg}$ ) at the depth of $12 \pm 3 \mathrm{~cm}$, as fixed by track data [38], points to the average pre-atmospheric radius of the chondrite of $R=48^{+12}-6 \mathrm{~cm}$ for the last $\sim 8$ years before entering the Earth atmosphere.

Knowing depth distributions of any cosmogenic radionuclide in a chondrite of any chemical type under irradiation by cosmic rays of any spectrum allows us to calculate its measured content at the time of the chondrite fall in any of its samples, and, therefore, to identify the size of the chondrite as well as the shelding depth of the sample. As shown in [35], apart from the analytical method, the calculation of the cosmogenic depth distribution in chondrites with the Monte Carlo approach also leads to the adequate estimates of sizes of the chondrites.

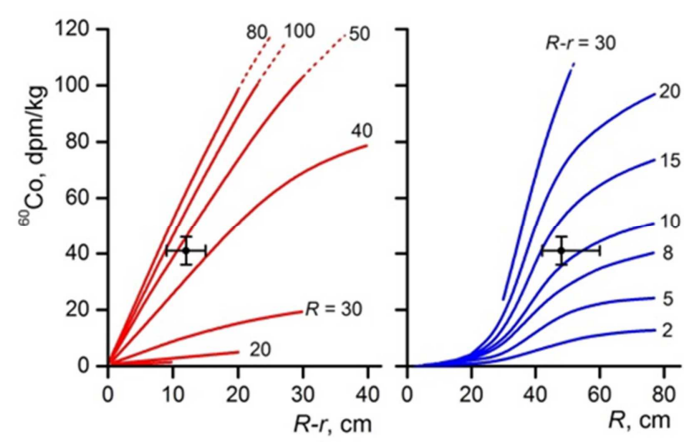

Figure 2. Simulation of the production rate of ${ }^{60} \mathrm{Co}$ in the Ash Creek chondrite [38]. Left: the ${ }^{60}$ Co distribution in L chondrites of radius $R$ depending on shielding depths $d=R-r$ of the samples; right: the ${ }^{60} \mathrm{Co}$ distribution at various shielding depths $d=R-r$ depending on the radius $R$. The cross shows the ${ }^{60}$ Co concentration measured in the chondrite $(41 \pm 5$ $\mathrm{dpm} / \mathrm{kg}$ ) at the shielding depth of $12 \pm 3 \mathrm{~cm}$ inferred from the track data. Numerals at the lines are the values of $R$ and $d=R-r$ (in cm). Both the plots indicate to the value of $R=48^{+12}{ }_{-6} \mathrm{~cm}$. 


\section{Monitoring of Cosmogenic Radionuclide Production Rates in the Chondrites Fallen in 1957-2016}

By nowadays, we have quantitatively analyzed the measured contents and calculated production rates of cosmogenic radionuclides at the time of fall of 42 chondrites in 1959-2016 (see their main parameters in the Table), which has resulted in a long set of homogeneous data on temporal and spatial variations of the cosmogenic radionuclide production rates at the heliocentric distances up to $\sim 5 \mathrm{AU}$ during $\sim 6$ solar cycles, demonstrating their monitoring [39]. In particular, in Figure 3 a long set of homogeneous data on variations of integral gradients of the ${ }^{54} \mathrm{Mn}$ and ${ }^{22} \mathrm{Na}$ production rates in the $19^{\text {th }}-24^{\text {th }}$ solar cycles, as well as on the average ${ }^{26} \mathrm{Al}$ production rates for the last million years, is presented. Further, in Figure $3 \mathrm{a}$ a special group of 10 chondrites, the falls of which onto the Earth were photographed, and thus, the parameters of their orbits are precisely calculated [2], is shown.

As it is clearly seen in Figure 3a, the data on ${ }^{22} \mathrm{Na}$ in the Lost City chondrite, on ${ }^{22} \mathrm{Na}$ and ${ }^{54} \mathrm{Mn}$ in Innisfree, on ${ }^{22} \mathrm{Na}$ in Moravka, as well as on ${ }^{22} \mathrm{Na}$ and ${ }^{54} \mathrm{Mn}$ in Kosice and on ${ }^{22} \mathrm{Na}$ in the Chelyabinsk chondrite, demonstrate the small (0$20) \% / \mathrm{AU}$ gradients of production rates of the radionuclides.
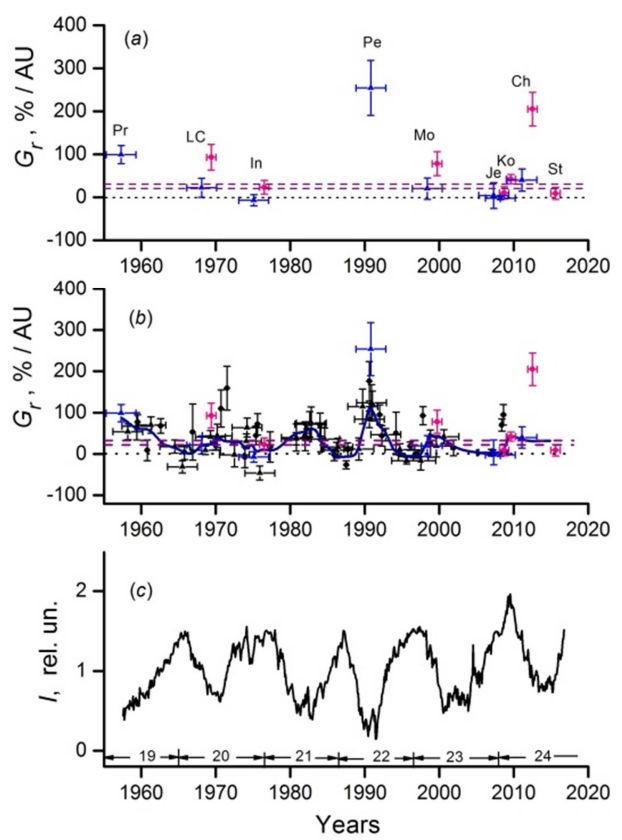

Figure 3. (a) Variations of integral gradients of the cosmogenic radionuclide production rates along the chondrite orbits (2-4 AU from the Sun) according to the measured contents of ${ }^{54} \mathrm{Mn}$ (red circles), ${ }^{22} \mathrm{Na}$ (blue triangles) and ${ }^{26} \mathrm{Al}$ (dash horizontal purple lines by 20-30\%/AU) in 10 chondrites with known orbits that fell between 1959 and 2016; (b) Variations of the integral gradients of cosmogenic radionuclide production rates along the chondrite orbits in 42 chondrites fallen in 1959-2016 (the curve is a polynomial smoothing curve of experimental data on five points taking into account the weight of each point); the unknown orbits of the chondrites are calculated by the phenomenological method (see Appendix); the notations are as in (a)). (c) Variations of GCR intensity ( $E>100 \mathrm{MeV})$ near the Earth according to stratospheric data [1].
Indeed, all the radionuclides in the chondrites were accumulated for the periods of minimum (or near the minimum) of the solar activity, when the GCR intensity along the chondrite orbits was as high as near the Earth (see the curve in Figure 3c). On the other hand, the data on ${ }^{22} \mathrm{Na}$ in the Pribram chondrite, on ${ }^{54} \mathrm{Mn}$ in Lost City, on ${ }^{22} \mathrm{Na}$ in Peekskill, as well as on ${ }^{54} \mathrm{Mn}$ in the Moravka and Chelyabinsk chondrites demonstrate much higher gradients of the radionuclide production rates, because the accumulation of radionuclides in those chondrites was going on during the time of the maximum (or near the maximum) of solar activity, under the strong decrease of the GCR intensity near the Earth registered by the stratospheric balloon experiments and shown in Figure 3c [1], as well as by the neutron monitor data [17].

Finally, the average gradients of $\sim 20-30 \%$ /AU for the contemporary solar cycles (according to ${ }^{22} \mathrm{Na}$ production rates) correspond to the average gradients for the last million years (according to ${ }^{26} \mathrm{Al}$ production rates; see the dashed horizontal lines in Figure 3), which testifies to the constancy of the mechanism of solar modulation, at least, over $\sim 1$ Myrs. These patterns were confirmed by our continuous study of cosmogenic radionuclides in other of 42 chondrites, the orbits of which are calculated by the phenomenological method (see Appendix).

The gradients of the radionuclide production rates in all the chondrites having fallen since 1959 are presented in Figure $3 \mathrm{~b}$. Please, pay attention to the fact that the analysis of each of ${ }^{54} \mathrm{Mn},{ }^{22} \mathrm{Na}$ and ${ }^{26} \mathrm{Al}$ radionuclides in each chondrite provides us with 3 values of gradients of their production rates at the respective heliocentric distances on the date of the chondrite fall. Therefore, analyzing the same radionuclides for the dates of fall of 42 chondrites of different orbits, we have 126 values of gradients of the radionuclide production rates at different heliocentric distances within the inner heliosphere.

The statistical smoothing of that continuum of homogeneous data (see the curve in Figure $3 b$ ) reveals the most general regularities:

1. Dependence of the radionuclide production rates on the phase of the solar cycle, i.e., their temporal variations.

2. Existence of gradients of the radionuclide production rates, i.e., their spatial variations along the chondrite orbits, namely, in the region of $\leq 5 \mathrm{AU}$ of the inner heliosphere, and also their dependence on the phase of solar activity.

3. Anticorrelation of the radionuclide production rates, as well as correlation of the magnitude of their gradients, with the development of solar activity.

4. Difference of variations of the gradients of the radionuclide production rates for various solar cycles, which might be conditioned by some peculiarities of the magneto hydrodynamic processes in the inner heliosphere.

5 . The average gradients of $\sim 20-30 \% / \mathrm{AU}$ for the contemporary solar cycles correspond to the average gradients for the last million years, which testifies to the 
constancy of the mechanism of solar modulation, at least, over $\sim 1$ Myrs.

The direct proportionality of the cosmogenic radionuclide production rates to the intensity of GCRs allows us to suggest the similar patterns in the cosmic rays that isotropically irradiated the Earth, as a minimum, on the way along their orbits, i.e., at the heliocentric distances of $\leq 5 \mathrm{AU}$ of the inner heliospere.

Table 1. List of 42 chondrites used in Figure 36 for monitoring variations of cosmogenic radionuclide production rates in the heliosphere in 1957-2016. The 10 chondrites with photographed orbits are marked with heavy-type [2]. The orbits of others are calculated by the phenomenological method (see Appendix). The chondrites with known atmospheric trajectories (visible radiants of falls) are marked by the asterisk [4].

\begin{tabular}{|c|c|c|c|c|c|c|}
\hline $\mathbf{N}$ & Meteorite & Type & Date of fall & Mass, kg & Q, A. U. & q, A. U. \\
\hline 1 & Pribram (Pr) & H5 & April 7, 1959 & 5.56 & 4.05 & 0.79 \\
\hline 2 & Bruderheim * & L6 & 4-Mar-60 & 303 & 4.06 & \\
\hline 3 & Harleton & L6 & 30-May-61 & 8.36 & 1.9 & \\
\hline 4 & Peace River * & L6 & 31-Mar-63 & 45.76 & 2.04 & \\
\hline 5 & Saint-Severin & LL6 & 27-Jun-66 & 271 & 1.97 & \\
\hline 6 & Denver & L6 & 15-Jul-67 & 0.23 & 2.1 & \\
\hline 7 & Lost City (LC) & H5 & 3-Jan-70 & 17 & 2.35 & 0.97 \\
\hline 8 & Malakal & L5 & 15 -Aug-70 & 2 & $\geq 4$ & \\
\hline 9 & Kabo & $\mathrm{H} 4$ & April 25, 1971 & 13.4 & 2.6 & \\
\hline 10 & Guibga & L5 & 26-Feb-72 & 0.288 & 2.1 & \\
\hline 11 & Gorlovka * & H3.7 & 17-Jul-74 & 3.62 & $\sim 1.9$ & \\
\hline 12 & Dhajala * & H3.8 & 28-Jan-76 & 45 & 2.25 & \\
\hline 13 & Jilin & H5 & 8-Mar-76 & 4000 & 2.17 & \\
\hline 14 & Innisfree (In) & L5 & 5-Feb-77 & 4.58 & 2.76 & 0.99 \\
\hline 15 & Kutais & H5 & 28-Nov-77 & 0.023 & 1.98 & \\
\hline 16 & Gujargaon* & H5 & 4-Sep-82 & 2.45 & $\leq 1.9$ & \\
\hline 17 & Wethersfield (1982) * & L6 & 8-Nov-82 & 2.76 & 2.37 & \\
\hline 18 & Tomiya * & $\mathrm{H} 4 / 5$ & 22-Aug-84 & 0.028 & 2.09 & \\
\hline 19 & Binningup * & H5 & $30-$ Sep- 84 & 0.488 & 1.81 & \\
\hline 20 & Kokubunji * & L6 & 29-Jul-86 & 11.51 & 1.93 & \\
\hline 21 & Trebbin & LL6 & 1-Mar-88 & 1.25 & 1.97 & \\
\hline 22 & Torino & H6 & 18-May-88 & 0.977 & 2.17 & \\
\hline 23 & Tahara & $\mathrm{H} 4 / 5$ & 26-Mar-91 & 1 & 1.92 & \\
\hline 24 & Noblesville & H4-6 & 31-Aug-91 & 0.484 & 1.92 & \\
\hline 25 & Mbale * & $\mathrm{L} 5 / 6$ & 14-Aug-92 & 150 & 2.51 & \\
\hline 26 & Peekskill (Pe) & H6 & 9-Oct-92 & 12.57 & 2.1 & 0.89 \\
\hline 27 & Mihonoseki * & L6 & 10-Dec-92 & 6.38 & 1.95 & \\
\hline 28 & Coleman * & L6 & 20-Oct-94 & 0.469 & 3.12 & \\
\hline 29 & Fermo & $\mathrm{H} 3-5$ & 25-Sep-96 & 10.2 & 1.97 & \\
\hline 30 & El Hammani * & H5 & 10-Aug-97 & $\sim 200$ & $\geq 4$ & \\
\hline 31 & Kunya-Urgench * & H5 & 20-Jun-98 & 1100 & $\sim 4$ & \\
\hline 32 & Hassi Labyade-NWA 820 & L3-5 & 15-Jun-99 & 2 & 1.9 & \\
\hline 33 & Moravka (Mo) & H5 & 6-Мay-00 & 0.633 & 2.71 & 0.99 \\
\hline 34 & Bukhara * & CV3 & 9-Jul-01 & 5.3 & 2 & \\
\hline 35 & Kilabo * & LL6 & 21-Jul-02 & 19 & 3.06 & \\
\hline 36 & Park Forest (PF) & $\mathbf{L 5}$ & 26-Mar-03 & 18 & 4.25 & 0.81 \\
\hline 37 & Tamdakht * & H5 & 20-Dec-08 & 100 & 3.54 & \\
\hline 38 & Ash Creek * & L6 & 15-Feb-09 & 9.5 & 3.99 & \\
\hline 39 & Jesenice (Je) & L6 & April 9, 2009 & 3.67 & 2.51 & 0.997 \\
\hline 40 & Košice (Ko) & H5 & 28-Feb-10 & 4.3 & 4.5 & 0.96 \\
\hline 41 & Chelyabinsk (Ch) & LL5 & 15-Feb-13 & 1000 & 2.78 & 0.74 \\
\hline 42 & Stubenberg (St) & LL6 & 6-Mar-16 & 1.47 & 2.14 & 0.918 \\
\hline
\end{tabular}

The probable errors of the calculated gradients of the radionuclide production rates are conditioned by many factors, and, according to the comprehensive analysis in [10], their accumulative values amount to $\geq 20 \%$, whereas the experimental errors of measured GCR gradients are about $10 \%$. For instance, in 1972-1975 at 2-3 AU the gradient of the ${ }^{22} \mathrm{Na}$ production rates with the GCRs $(E>100 \mathrm{MeV})$ in the Dhajala chondrite was $61 \pm 28 \% / \mathrm{AU}$ [40], and the measured gradient of protons of $E>70 \mathrm{MeV}$ during the flights of Pioneer 10 and 11 at the heliocentric distances $<5$ AU in 1972-1974 was averaged as 3.4 $\pm 0.4 \% / \mathrm{AU}$ [20]. This means, that the gradients of the radionuclide production rates are not just the GCR gradients, as well as they do not equal each other - differing, as a minimum, by an order of magnitude. Meanwhile, the magnitudes of the cosmogenic radionuclide production rates are conditioned by the GCR intensity, so that the revealed regularities of their temporal and spatial variations demonstrate the existing similar regularities of the GCR variations in the inner heliosphere over the long-time scale.

Even the first superficial consideration of the patterns makes it possible to notice some differences of the GCR temporal and spatial variations in the different solar cycles, which stimulates the further study of features and peculiarities of the inner heliosphere. 


\section{Correlative Analysis of Processes in the Inner Heliosphere}

The multi-year studies of processes of solar modulation of GCRs in the heliosphere have led to the great progress in this field, as well as to the better understanding of the extremely high complexity of the problem [14]. At the same time, the appropriate ways of further development and perfection of the investigation have become clear [41]. The extensive and comprehensive studies of processes in the inner heliosphere provide long sets of homogeneous data on many important parameters and their variations, which allow us to investigate the dynamics of the processes and the structure of the heliosphere. The invaluable databases of free use on the solar activity (SA) [http://www.sidc.be/silso/DATA/yearssn.data], on the strength B of the interplanetary magnetic fields (IMF) [http://nssdc.gsfc.nasa.gov/omniweb/form/dx1.html], on the tilt angle $\alpha$ of the heliospheric current sheet (HCS) [http://wso.stanford.edu/Tilts.html], on the total solar magnetic field (TSMF) inversions in WSO Polar Field Observation (1976-present)) [http://wso.stanford.edu ], etc., in combination with the multi-year data of permanent measurements of integral GCR intensity in different energy ranges by using neutron monitors and other ground-based and underground equipments or stratospheric balloon and space IMP experiments, enable us to study the comprehensive causal relationships of processes on the Earth and in the Solar system, deriving the most general and important regularities (see it below in detail). Moreover, the investigation of correlations between the parameters of different processes provides the possibilities not only to reproduce various processes in the past, but also to forecast their development in the nearest future [42-44]. Such an approach is very useful for studying many natural processes on the Earth, including climate variations [42, 45].

All the above available information concerns the processes near the Earth, i.e., at $\sim 1 \mathrm{AU}$. What is happening at further heliocentric distances of the inner heliosphere? The direct GCR intensity measurements in that region are very scarce and, besides, they belong to different periods, [19-21, 23], so that they might not constitute an adequate dynamic sequence and interrelation of processes in that range of the heliosphere. Just such a pattern provides the monitoring of gradients of the cosmogenic radionuclide production rates in the chondrites of different orbits and various dates of fall in 1959-2016 (Figure 3b), which allows us to carry out the correlative analysis of operative processes in the inner heliosphere. In Figure 4, a set of homogeneous data on gradients of the cosmogenic radionuclide production rates in 42 chondrites (curve 1 from Figure $3 b$ ) as compared with variations of the solar activity (Wolf numbers $\mathrm{Rj}$; curve 2) [46], the strength $\mathrm{B}$ of the interplanetary magnetic field (curve 3) [47] and the tilt angle $\alpha$ of the heliospheric current sheet (curve 4) [48] for 1957-2016 are presented.

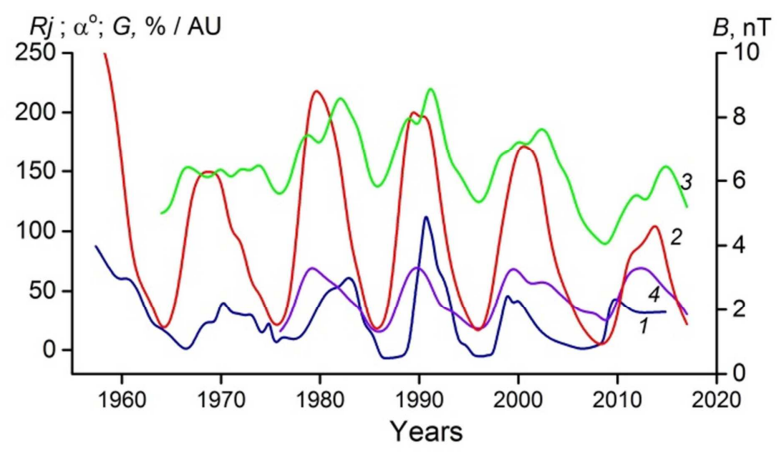

Figure 4. Temporal and spatial variations of the gradients of the cosmogenic radionucide production rates (curve 1 from Figure $3 b$ ) in comparison with variations of the solar activity (Wolf numbers Rj; curve 2), strength $B$ of the interplanetary magnetic field (curve 3) and tilt angle $\alpha$ of the heliospheric current sheet (curve 4) for 1957-2016 [46-48] are demonstrated.

As a general result, the positive correlation of all the operative factors should be noted, which points out to the definite role of the solar activity in the range of the inner heliosphere [49]. However, as it is well seen in Figure 4, there is a difference of correlations for the different solar cycles, in particular, some difference of correlations for the phases of growth and decline of the solar cycles, which might be conditioned by the stochastic nature of developing solar activity.

Indeed, at the heart of the free rotation of the solar dynamo, there are turbulent convection of the solar plasma and its differential rotation [50]. When some conditions of the convection generation are disturbed, or the interaction between convection and differential rotation is disturbed (e.g., because of dynamical viscosity (intrinsic friction)), then some states of instability arise, the output ambiguity from which leads to some disturbance of the solar dynamo operation.

The most apparent stochastic processes take place at the moments of polarity inversion of the solar magnetic fields at each maximum of the solar activity, which leads to the change of the 22-year magnetic cycles. The inversion can begin and end at any time, and it may be different in the Nand S-hemispheres of the Sun, which sometimes leads to the periods when both the poles turn out to have the same polarity (http://wso.stanford.edu/Polar.html) [51, 52]. Thus, in the $20^{\text {th }}$ solar cycle the polarity of both the poles was negative approximately for a year since August 1970, i.e., the heliosphere turned out to be open for the positively charged particles [53]. That resulted in the additional drift of the GCR protons into the Solar system and, in general, in the higher GCR intensity level near the Earth [1], as well as to the small gradients of cosmogenic radionuclide production rates in the chondrites, having fallen in the $20^{\text {th }}$ solar cycle, in comparison with the $19^{\text {th }}$ one [54] (see. Figure 3).

Quite a reverse situation was observed during the maxima of the $22^{\text {nd }}$ and $24^{\text {th }}$ solar cycles, when the polarity of both the poles was positive, so that the heliosphere was closed for the positively charged particles. That led to the deepest minimum 
of the GCR intensity in the stratosphere in 1990-1991, as well as to the highest gradients of the cosmogenic radionuclide production rates in the chondrites that fell during that time period $[1,54]$ (see. Figure 3).

The situation at the maximum of the $24^{\text {th }}$ solar cycle is especially interesting [55]. The observed weakness of the magnetic fields, as well as the unusual duration of the solar activity decrease before the $24^{\text {th }}$ solar cycle resulted, in general, in higher, GCR intensity near the Earth, as well as in small, on the average, gradients of the cosmogenic radionuclide production rates in several chondrites that fell for that time. The period when both the poles were positive proceeded for 14 months only (from June 2012 up to July 2013). However, that period coincided with the ${ }^{54} \mathrm{Mn}$ accumulation period (from December 2011 up to February 2013) before the Chelyabinsk chondrite fall onto the Earth, which resulted in the high gradient of its production rate in this chondrite, whereas the gradient of ${ }^{22} \mathrm{Na}$ production rate for 2008-2013 in the Chelyabinsk is similar to other data for that time (see Figure 3) [10, 35, 39].

The obtained result demonstrates the utmost sensitivity of the cosmogenic radionuclide production rates in chondrites to the smallest changes of the magneto hydrodynamic conditions in the heliosphere, which provides us with a highprecision method for studying processes of the GCR solar modulation at any heliocentric distances of the inner heliosphere over a long-time scale $[39,56]$.

\section{Conclusions and Further Perspectives}

Meteorites provide a comparatively cheap method, and (with the recent and more frequently photographed falls) they bring the unique information on the peculiarities of the solar modulation and other dynamic processes in the heliosphere, as well as on the structure of the heliosphere itself. The gradients of cosmogenic radionuclide production rates, estimated over a long-time scale at different heliocentric distances, provide the possibility of reconstructing the regularity in the past as well as of forecasting the radiation situation in the interplanetary space, which is very important for prospective cosmic manned flights.

Certainly, the progress in the low-level counting, in the perfection of the theoretical approaches to the analysis of cosmogenic radionuclides in cosmic bodies, in the permanent work of the world bolide's net for photographing meteoritic falls, etc., over the last half-century leads to the higher precision of the results, whereas the work of many specialists using rather different approaches has enlarged the amount of the independent data, i.e. has improved the statistics of the results.

In other words, the measurement and theoretical analysis of cosmogenic radionuclides in consecutively fallen chondrites could provide a unique space-time continuum of the cosmogenic radionuclide production rates and their variation over a long-time scale, which might be useful in the correlative analysis of operative processes in the inner heliosphere.
Indeed, precise measurements of the time-dependent intensity of the GCRs at $1 \mathrm{AU}$ in the PAMELA experiments make it possible to elaborate numerical models, which-taking into account the observed peculiarities of the processes during Ulysses and other spacecraft missions, or adopting the modified Parker heliospheric magnetic field with variable diffusion coefficients-develop some real pictures of the threedimensional propagation of GCRs through the heliosphere, e.g. [57]. It is clear that just the behavior of cosmogenic radionuclide production rates, which demonstrates the real temporal and spatial distribution of GCRs in the inner heliosphere is the only experimental criterion of validity of such models, i.e. at present, the experimental study of the solar modulation of GCRs in the inner heliosphere by using cosmogenic radionuclides in the chondrites permanently falling to the Earth has no alternative.

However, such an investigation is based on the data of many specialists in some joint fields and, in order to be efficient, demands foundation of the International Consortium of the Meteorite Community for organization of an International Meteorite Patrol Service for the comprehensive experimental and theoretical study of cosmogenic radionuclides in chondrites, permanently falling onto the Earth, to investigate the distribution and dynamics of GCRs in the inner heliosphere.

The early delivery of fresh-fallen chondrites to laboratories in order to catch the short-lived radionuclides, at least, ${ }^{54} \mathrm{Mn}$ and ${ }^{22} \mathrm{Na}$, for the successive radionuclide studies is the important prerequisite for future investigations using meteorites as probes of GCRs.

\section{Appendix: Phenomenological Approach for Estimation of Extension of Chondrite Orbits}

Starting from the first measurements of ${ }^{26} \mathrm{Al}$ in the chondrites Pribram, Lost City and Innisfree, which were further supported by the data for other chondrites of known orbits, we have found that there exists a $20-30 \% / \mathrm{AU}$ gradient of ${ }^{26} \mathrm{Al}$ production rate along the chondrite orbits (at least $<5$ $\mathrm{AU})$, which is demonstrated in Figure 5 [9, 58, 59].

Such an experimental profile of the ${ }^{26} \mathrm{Al}$ production rate dependence on the heliocentric distance may be approximated (within the error) by a broken line, describing the "minimal" ${ }^{26} \mathrm{Al}$ production rate $\left(H_{\mathrm{min}}\right)$ under irradiation by the average GCR spectrum over the solar cycle (average spectrum of 1962) up to $1.9 \mathrm{AU}$ and the maximum one $\left(H_{\max }\right)$ under irradiation by the practically unmodulated GCR spectrum in April-May of 1965 at further distances. Then the measured $\left(H_{\mathrm{msd}}\right)$ content of ${ }^{26} \mathrm{Al}$ may be expressed as

$$
H_{m s d}=H_{\min } Z+H_{\max }(1-Z)
$$

where $Z$ is a time interval (in units of orbital period $T=1$ ) for which the chondrite flies in the range before $\sim 1.9$ AU.

The regularity (8) is used in the "isotopic" approach for 
estimating the position of aphelia $\mathrm{Q}$ of the chondrites, in accordance with their ${ }^{26} \mathrm{Al}$ content $[9,58,59]$

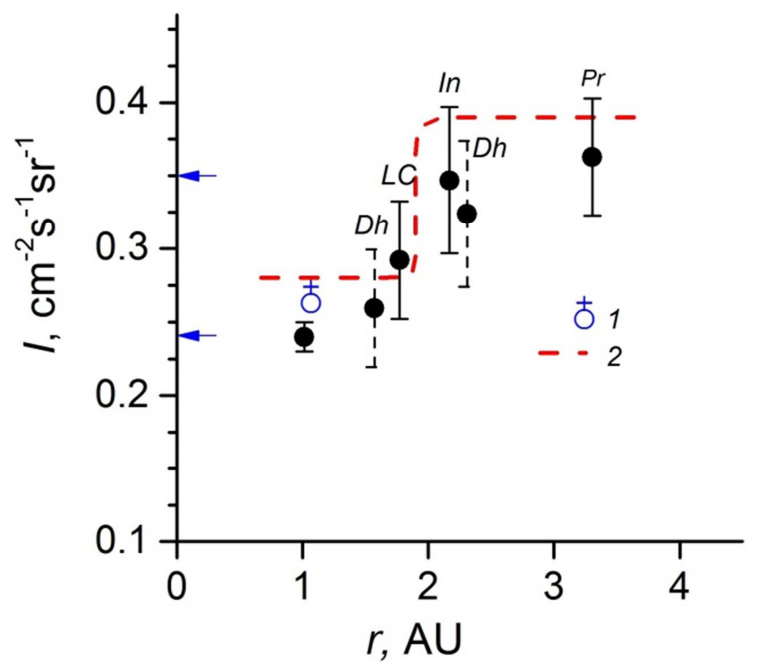

Figure 5. Spatial increase of the GCR (E>100 MeV) intensity, averaged for $\sim 1 \mathrm{Myr}$, according to the ${ }^{26}$ Al content in chondrites. 1-near the Earth (due to $26^{A l}$ in lunar samples); 2-approximation line with the average GCR intensity for the solar cycle up to $\sim 2 A U$ and for the unmodulated GCR intensity beyond $\sim 2 A U$.

An effective phenomenological expression $Q(Z)$ is derived using the general equation of the second order curve (describing the motion of the celestial bodies):

$$
a Z^{2}+2 b Z Q+c Q^{2}+2 d Z+2 e Q+f=0
$$

at the boundary conditions: $\mathrm{Z}=1$ if $\mathrm{Q}=1.9 \mathrm{AU} ; \mathrm{Z}=0$ if $\mathrm{Q} \sim$ $\infty$ (for practically parabolic orbits). The coefficients in this equation are determined by using the parameters of known orbits, e.g. $\mathrm{LC}(\mathrm{Z}=0.5 ; \mathrm{Q}=2.35 \mathrm{AU}), \operatorname{Pr}(\mathrm{Z}=0.19 ; \mathrm{Q}=4.05$ $\mathrm{AU})$, etc. As a result, the following simple expression is obtained:

$$
Q(Z)=1.25+0.13 Z+0.53 Z^{-1}
$$

where $\mathrm{Q}$ is the aphelion in AU. Deriving Z from (8), one may estimate $\mathrm{Q}$ of a chondrite, if its ${ }^{26} \mathrm{Al}$ content is available. The properties, the range of applicability and errors of the approach are discussed in detail in $[9,58,59]$. The "isotopic approach" is easy to use. It was studied and verified in detail by [60], who confirmed its efficiency.

The largest probability to fall to the Earth and become meteorites belongs to celestial objects that have the perihelion $\mathrm{q} \sim 1 \mathrm{AU}$. This follows from the data of visible radiants of meteorite falls, as well as from the thermoluminescence of chondrites: $\mathrm{q} \sim 0.8-1.0 \mathrm{AU}$ [3]. Within such an approximation, the most probable values of $a$ and $e$ are as follows:

$$
a \sim \frac{q+1}{2}, \quad e \sim \frac{q-1}{q+1}
$$

and the knowledge of these values permits us to estimate approximately the orbit of any chondrite.

\section{Acknowledgements}

We are grateful to professor Bazilevskaya Galina Aleksandrovna (bazilevs@sci.lebedev.ru) and all her colleagues in the Lebedev Physical Institute, Russian Academy of Sciences for the permission to use their long sequences of homogeneous data on the GCR intensity in the stratosphere.

This work was supported in part by the Program No. 12 of Fundamental Research of Russian Academy of Sciences.

\section{References}

[1] Stozhkov, Yu. I., Svirzhevsky, N. S., Bazilevskaya, G. A., Kvashnin, A. N., Makhmutov, V. S., and Svirzhevskaya, A. K. 2009. "Long-term (50 years) measurements of cosmic ray fluxes in the atmosphere". Adv. Space Res. 44 (10). 11241137.

[2] Meier M. M. M. 2016. "Meteorites with photographic orbits" http://www.meteoriteorbits.info.

[3] Benoit, P. H., Sears, D. W. G., and McKeever, S. W. S., 1991. "The Natural Thermoluminescence of Meteorites. II. Meteorite Orbits and Orbital Evolution”. Icarus, 94, 311-325.

[4] Levin B. Yu., and Simonenko A. N. 1969. "Meteorute radiants and orbits". Meteorite Research. Reidel. Dordrecht. 552-558.

[5] Bhandari, N., Lal, D., Rajan, R. S., Arnold, J. R., Marti, K., and Moor, C. B. 1980. "Atmospheric ablation in meteorites: A study based on cosmic ray tracks and neon isotopes". Nucl. Tracks, 4 (4), 213-262.

[6] Millman P. M. 1969. "Astronomical information on meteorite orbits". Meteorite Research. Reidel. Dordrecht. 541-551.

[7] Wetherill G. W. 1969. "Relationships between orbits and sources of chondritic meteorites". Meteorite Research. Dordrecht. 573-589.

[8] Landau L. D. and Lifshitz E. M. 1958. Mekhanika. Fizmatgiz Moscow: 1958, pp. 49-54. [Mechanics. Course of Theoretical Physics vol. 1. Pergamon Press. 1960].

[9] Lavrukhina A. K., and Ustinova, G. K. 1990. Meteorites as Probes of Cosmic Ray Variations. Nauka, Moscow (in Russian) 262p.

[10] Alexeev V., Laubenstein M, Povinec P. P., and Ustinova G K. 2019. "Cosmogenic radionuclides in meteorites and solar modulation of galactic cosmic rays in the internal heliosphere". Solar System Research. 53 (2). 98-115.

[11] Jull A. J. T., and Burr G. S. 2013. "Mass Spectrometry Instruments VI: Accelerator Mass Spectrometry." Treatise of Geochemistry (eds. K. K. Turekian and H. Holland): Elsevier. Amsterdam. 15. 375-383.

[12] Laubenstein M., Hult M., Gasparro J., and Arnold D., Neumaier S., Heusser G., Köhler M., Povinec P. P., Reyss J.-L., Schwaiger M., Theodorsson P. 2004. „Underground measurements of radioactivity”. Appl. Rad. Isotopes. 61. 167-172.

[13] Povinec P. P., Comanducci J. F., and Levy-Palomo I. 2005. "IAEA-MEL's underground counting laboratory (CAVE) for the analysis of radionuclides at very low-levels". J. Radioanal. Nucl. Chem. 263. 441-445. 
[14] Potgieter M. S. 2013. "Solar modulation of cosmic rays". Living Rev. Solar Phys. 10. 3-66. ArXiv: 1306.4421 v1[physics.space-ph] 19 June 2013; doi: 10.12942/lrsp-2013.

[15] Parker E. N. 1979. Cosmic magnetic fields. Clarendon press. Oxford. 841p.

[16] Burlaga L. F. and Ness N. F. 1998. "Magnetic field strength distributions and spectra in the heliosphere and their significance for cosmic ray modulation: Voyager 1, 19801994”. J. Geophys. Res. 103. 29719-29732.

[17] Moraal, H., and Stoker, P. H. 2010. "Long-term neutron monitor observations and the 2009 cosmic ray maximum." $J$. Geophys. Res. 115 (A12). doi 10.1029/2010JAO15413.

[18] Galper A., and Spillantini P. 2017. "Ten Years of CR Physics with PAMELA." Physics of Particles and Nuclei V. 48, No. 5, pp. 710-719.

[19] O'Gallagher J. J., and Simpson J. A. 1967. "The heliocentric intensity gradient of cosmic ray protons and helium during minimum solar modulation." Astrophys. J. 147 (2), 819-827.

[20] McKibben R. B., O’Gallagher J. J., Pyle K. R., and Simpson J. A. 1977. "Cosmic ray intensity gradients in the outer solar system measured by Pioneer 10 and 11." Proc. $15^{\text {th }}$ Intern. Cosm. Ray Conf. Plovdiv. 3. 240-245.

[21] Venkatesan D., Decker R. B., and Krimigis S. M. 1987. "Cosmic ray intensity gradients during 1984-1986." Proc. $20^{\text {th }}$ Intern. Cosm. Ray Conf., Moscow. 3. 385-388.

[22] Potgieter M. S., and Le Roux J. A. 1987. "On a possible modulation barrier in the outer heliosphere." Proc $20^{\text {th }}$ Intern. Cosm. Ray Conf, Moscow. 3. 291.

[23] McKibben R. B., Connell, Lopate, C., Zhang, M., Anglin, J. D., Balogh, A., Dalla, S., Sanderson, T. R., Marsden, R. G., Hofer, M. Y., Kunow, H., Posner, A., and Heber, B. 2003. "Ulysses COSPIN observations of cosmic rays and solar energetic particles from the South Pole to the North Pole of the Sun during solar maximum". Ann. Geophys. 21. 1217-1228.

[24] Belov A. V., Eroshenko E. A., Heber B., Yanke V. G., Raviart A., Mueller-Mellin R., Kunow H., Roehrs K., Wibberenz G., Paizis C. 2001. "Latitudinal and radial variation of $>2 \mathrm{GeV} / \mathrm{n}$ protons and $\alpha$-particles in the southern heliosphere at solar maximum: ULYSSES COSPIN/KET and neutron monitor network observations". Proc. 27 $7^{\text {th }}$ Intern. Cosm. Ray Conf., Hamburg, 10, 3996-3999.

[25] Lavrukhina A. K., and Ustinova, G. K. 1971. "Solar proton medium flux constancy over a million years". Nature. 232 (5311). 462-463. doi: 10.1038/232462a0.

[26] Usoskin I. G., Desorgher L., Velinov P., Storini M, Flückiger E. O., Bütikofer R., and Kovaltsov G. A. 2009. "Ionization of the Earth's atmosphere by solar and galactic cosmic rays." Acta Geophys. 57 (1), 88-101. DOI: 10.2478/s11600-008-0019-9.

[27] Lavrukhina A. K., and Ustinova, G. K. 1978. "On the absence of effective modulation of galactic cosmic rays in the solar system during the ice-age." Proc. $9^{\text {th }}$ Lunar Planet. Sci. Conf. LPI. Houston. 2. 2399-2414.

[28] Lavrukhina A. K. and Ustinova, G. K. 1990b.” Depth distribution regularities of cosmogenic radionuclides in meteorites". Proc. $21^{\text {st }}$ Intern. Cosm. Ray Conf. Adelaida. 7. 145-148.
[29] Ustinova G. K. and Lavrukhina A. K. 1990. "Analytical Expressions for Distribution of Cosmic Radiation and Radionuclides in Meteorites", Proc. $21^{\text {st }}$ Intern. Cosm. Ray Conf., Adelaide. 7. 141-144.

[30] Ustinova G. K., and Lavrukhina, A. K. 1993. "On modeling nuclear reactions in meteorites". Lunar Planet. Sci. Conf. $24^{\text {th }}$. LPI. Houston. Part 3. 1457-1458.

[31] Lavrukhina, A. K., Ustinova, G. K., Malyshev, V. V., and Satarova, L. M. 1973. "Modeling nuclear reactions in an isotropically irradiated thick target." Soviet Atomic Energy 34 (1). 29-35.

[32] Ustinova, G. K., Alexeev, V. A., and Lavrukhina, A. K. 1989. "Methods of determining sizes of meteorites before atmospheric entry." Geochem. Intern. 26 (5). 1-16.

[33] Eberhardt P., Geiss J., and Lutz H. 1963. "Neutrons in meteorites." Earth Science and Meteoritics. North-Holland. Amsterdam. 143-168.

[34] Povinec, P., Masarik, J., Sýkora, I., Kováčik, A., Beňo, J., Meier, M. M. M., Wieler, R., Laubenstein, M., and Porubčan, V. 2015. "Cosmogenic radionuclides in the Košice meteorite: Experimental investigations and Monte Carlo simulations". Meteoritics \& Planetary Science. 50. 880-892. doi: 10.1111/maps. 12380 .

[35] Alexeev V. A., Laubenstein M., Povinec P. P., and Ustinova G. K. 2015. "Variations of cosmogenic radionuclide production rates along the meteorite orbits". Adv. Space Res. 56. 766-771.

[36] Bhattacharya S. K. Goswami, J. N., and Lal, D. 1973. "Semiempirical rates of formation of cosmic ray tracks in spherical objects exposed in space: Pre-and post-atmospheric depth profiles". J. Geophys. Res. 78 (34), 8356-8363.

[37] Jarosewich E. 1990. "Chemical analyses of meteorites: A compilation of stony and iron meteorite analyses". Meteoritics. 25. 323-337.

[38] Alexeev, V. A., Gorin, V. D., Ivliev, A. I., Kashkarov, L. L., Ott, U., Sadilenko, D. A., and Ustinova, G. K. 2012. "Integrated Study of the Thermoluminescence, Noble Gases, Tracks, and Radionuclides in the Fresh Fallen Ash Creek L6 and Tamdakht H5 Chondrites." Geochemistry International. 50 (2). 105-124.

[39] Ustinova G. K., and Alexeev V. A. 2019. "Variations of Cosmogenic Radionuclide Production Rates in Chondrites of Known Orbits". Doklady Physics. 64 (3). 139-143. doi: $0.1134 / \mathrm{S} 1028335819030029$.

[40] Lavrukhina A. K., and Ustinova G. K. 1981. "Galactic cosmic-ray gradients in the ecliptic cycles (meteorite data)". Adv. Space Res. 1 (3). 143-146.

[41] Mewaldt R. A., 2013. "Cosmic rays in the heliosphere: Requirements for future observations”. Space Sci. Rev. 176. 365-390; doi: 10.1007/s11214-012-9922-0.

[42] Ahluwalia, H. S. 2014. "Sunspot activity and cosmic ray modulation at 1 a. u. for 1900-2013". Adv. Space. Res. 54 (8), 1704-1716, 2014; doi: 10.1016/j.asr.2014.06.034.

[43] Ahluwalia, H. S., and Jackiewicz J, "Sunspot cycle 23 descent to an unusual minimum and forecasts for cycle 24 activity", Adv Space Res. 50, 662-668, 2012; doi: 10.1016/j.asr.2011. 04.023, 2012. 
[44] Okhlopkov V. P., and Stozhkov Yu. I. 2011. "Solar activity at present and in the near future". Bull. Russ. Acad. Sci. Phys. 75 (6), 860-863.

[45] Alexeev, V. A. 2007. "Some features of climate change on Earth and its possible relation to solar-activity variations." Solar System Res. 41 (6), 527-534, doi: 10.1134/S0038094607060093.

[46] http://www.sidc.be/silso/DATA/yearssn.data.

[47] http://nssdc.gsfc.nasa.gov/omniweb/form/dx1.html.

[48] http://wso.stanford.edu/Tilts.html.

[49] Miroshnichenko Leonty. 2015. Solar Cosmic Rays. Fundamentals and Applications. Springer. 456p.

[50] Zeldovich, Ya. B., and Ruzmaikin, A. A., Sokoloff, D. D. 1983. Magnetic Fields in Astrophysics. Gordon and Breach, New York. 382p.

[51] http://wso.stanford.edu.

[52] http://wso.stanford.edu/Polar.html.

[53] Ustinova, G. K. 1983. "Quasistationary asymmetry of the GCR density distribution in the heliosphere". Proc. $18^{\text {th }}$ Intern Cosm. Ray Conf. Bangalore. 10. 71-74.
[54] Alexeev, V. A., and Ustinova, G. K. 2006. "Solar modulation of galactic cosmic rays in the three-dimensional heliosphere according to meteorite data." Geochem. Intern. 44, 423-438.

[55] Ishkov V. N. 2010. "Properties and Surprises of Solar Activity XXIII Cycle." Sun and Geosphere. 5 (2), 43-46.

[56] Ustinova G. K. 2016. "Patterns of Cosmogenic Radionuclide Production Rates in the Heliosphere and Problems of Solar Modulation on a Long Time Scale." Doklady Physics. 61 (11). 571-575.

[57] Qin G., and Shen Z.-N. 2017. "Modulation of Galactic Cosmic Rays in the Inner Heliosphere, Comparing with PAMELA Measurements." The Astrophysical Journal, 846: 56 (11pp), 2017 September 1 https://doi.org/10.3847/15384357/aa83ad. The American Astronomical Society.

[58] Lavrukhina A. K., and Ustinova G. K., 1972. "Cosmogenic radionuclides in stones and meteorite orbits". Earth and Planet. Sci. Lett. 15 (4). 347-360.

[59] Ustinova G., and Lavrukhina A. K., 1980. "Phenomenological expression for estimation of aphelia of fallen meteorites." Lunar Planet. Sci. XI. Houston. LPSI. 1187-1189.

[60] Cameron J. R., and Top Z. 1974. "Measurement of ${ }^{26} \mathrm{Al}$ in stone meteorites and its use in the derivation of orbital elements." Geochim. Cosmochim. Acta. 38 (6). 899-909. 